\title{
The Unexpected and Exceptionally Facile Chemical Modification of the Phenolic Hydroxyl Group of Tyrosine by Polyhalogenated Quinones under Physiological Conditions
}

\author{
$\mathrm{Na} \mathrm{Qu}^{\dagger}{ }^{\dagger}$ Feng Li, ${ }^{\dagger}$ Bo Shao, ${ }^{\dagger}$ Jie Shao, ${ }^{\dagger}$ Guijin Zhai, ${ }^{\ddagger}$, Fuyi Wang, ${ }^{\ddagger}$ and Ben-Zhan Zhu*, ${ }^{\dagger}$
}

${ }^{\dagger}$ State Key Laboratory of Environmental Chemistry and Eco-toxicology, Research Centre for Eco-environmental Sciences and University of the Chinese Academy of Sciences, the Chinese Academy of Sciences, 18 Shuangqin Road, Haidian District, Beijing 100085, P. R. China

${ }^{\ddagger}$ Beijing National Laboratory for Molecular Sciences, CAS Key Laboratory of Analytical Chemistry for Living Biosystems, Institute of Chemistry, Chinese Academy of Sciences, Beijing 100190, P. R. China

${ }^{\S}$ Tianjin Research Centre of Basic Medical Sciences, Tianjin Medical University, Tianjin 300070, P. R. China

\section{Supporting Information}

ABSTRACT: The phenolic hydroxyl group of tyrosine residue plays a crucial role in the structure and function of many proteins. However, little study has been reported about its modification by chemical agents under physiological conditions. In this study, we found, unexpectedly, that the phenolic hydroxyl group of tyrosine can be rapidly and efficiently modified by tetrafluoro-1,4-benzoquinone and other polyhalogenated quinones, which are the major genotoxic and carcinogenic quinoid metabolites of polyhalogenated aromatic compounds. The modification was found to be mainly due to the formation of a variety of fluoroquinone-O-tyrosine conjugates and their hydroxylated derivatives via nucleophilic substitution pathway. Analogous modifications were observed

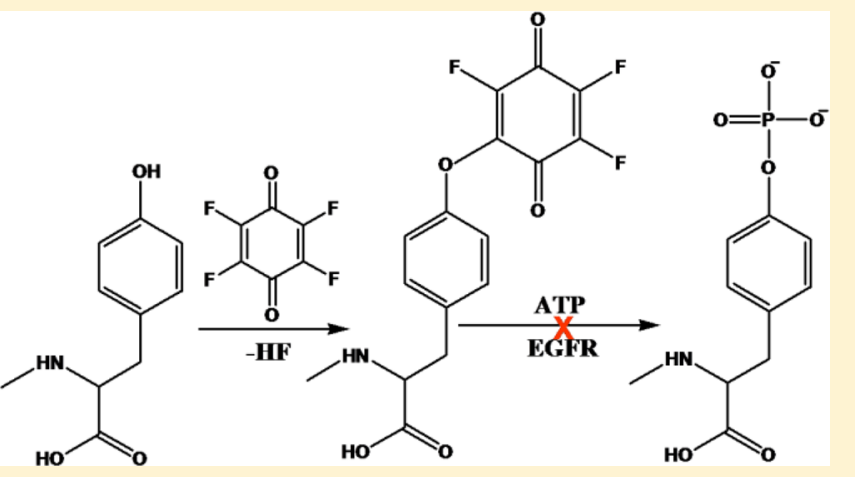
for tyrosine-containing peptides. Further studies showed that the blockade of the reactive phenolic hydroxyl group of tyrosine in the substrate peptide, even by very low concentration of tetrafluoro-1,4-benzoquinone, can prevent the kinase catalyzed tyrosine phosphorylation. This is the first report showing the exceptionally facile chemical modification of the phenolic hydroxyl group of tyrosine by polyhalogenated quinones under normal physiological conditions, which may have potential biological and toxicological implications.

\section{INTRODUCTION}

The phenol moiety of tyrosine residues plays an important functional role in protein post-translational modifications (PTMs) such as protein tyrosine phosphorylation, nitration, and $\mathrm{O}$-sulfation, which are involved in numerous physiological and pathological processes. ${ }^{1-3}$ Protein tyrosine phosphorylation is considered to be one of the key steps in signal transduction and associated with cell proliferation, survival, apoptosis, mobility, and adhesion. Due to its redox activity, the phenolic hydroxyl group of tyrosine is the active site of many enzymes such as ferredoxin- $\mathrm{NADP}^{+}$reductase and DNA topoisomerase. $^{4-6}$

Chemical modification of tyrosine can be achieved by nucleophilic substitution at the hydroxyl group. N-Acetylimidazole is the most commonly used nucleophilic reagent to modify phenolic hydroxyl groups, and can acetylate tyrosyl residues under $\mathrm{pH} 7.5$ and room temperature with a high molar ratio. ${ }^{7}$ Acylation of tyrosine is accompanied by distinctive spectral changes and easy to be detected.
Tetrahalo-1,4-benzoquinones (TXBQs) are the major genotoxic and carcinogenic quinoid metabolites of the widely used biocide pentahalophenols (PXPs). ${ }^{8-10}$ TXBQs have also been found as reactive oxidation intermediates or products in processes employed to oxidize or remediate PXPs and other polychlorinated persistent organic pollutants (POPs) in various enzymatic and chemical systems. ${ }^{11,12}$ The parent quinone is known for high chemical reactivity with free thiol groups of cysteine residues in proteins. Macromolecular adducts of tetrachloro-1,4-benzoquinone (TCBQ) with cysteinyl residues of the blood proteins have been characterized in vivo. ${ }^{13}$

During our study on the mechanism of genotoxicity for polyhalogenated phenols and their quinoid metabolites, we found that the highly reactive hydroxyl radicals $\left(\mathrm{HO}^{\bullet}\right)$ can be produced by halogenated quinones and $\mathrm{H}_{2} \mathrm{O}_{2}$ independent of transition metal ions, which can cause DNA damage and lipid peroxidation. ${ }^{14-19}$ Compared with the classic metal-mediated

Received: June 18, 2016

Published: September 9, 2016 
inorganic Fenton system, the newly discovered metalindependent $\mathrm{HO}^{\bullet}$ producing system $\left(\mathrm{TXBQ} / \mathrm{H}_{2} \mathrm{O}_{2}\right.$ ) can be regarded as a novel organic Fenton-like system.

In a recent study, an electrochemical biosensor was fabricated and used to detect tyrosine oxidation induced by classic Fenton reagents $\left(\mathrm{Fe}(\mathrm{II}) / \mathrm{H}_{2} \mathrm{O}_{2}\right) .{ }^{20}$ We found that tyrosine in peptide was oxidized and the electrochemical signal of the peptide decreased markedly after incubation with Fenton reagents. When we extended our studies from the classic iron-mediated Fenton system to the new TXBQ-mediated organic Fenton-like system, we expected that the results should be similar.

However, unlike in Fenton system and to our great surprise, we found that TXBQs alone can cause a dramatic decrease of electrochemical signal of peptide, which is even stronger than the combination of TXBQ and $\mathrm{H}_{2} \mathrm{O}_{2}$ together. Interestingly, we found that the underlying mechanism is due to an exceptionally facile chemical modification of the phenolic hydroxyl group of tyrosine by TXBQs.

\section{MATERIALS AND METHODS}

L-Tyrosine was obtained from Shanghai Biochemicals (Shanghai, China). Poly(diallyldimethylammonium chloride) (PDDA), tetrafluoro-1,4-benzoquinone (TFBQ), tetrachloro-1,4-benzoquinone (TCBQ) and poly(glu, tyr) (4:1) sodium salt, epidermal growth factor receptor (EGFR), EGF, and adenosine triphosphate (ATP) were obtained from Sigma-Aldrich (St. Louis, MO, USA) and used as received. EEEEEY and AGYTAIVSHR (purity: 98\%) were synthesized by Invitrogen (Carlsbad, California, USA). RRLIEDNEYTARG (substrate peptide), RRLIEDNEpYTARG (phosphorylated substrate peptide), and internal standard RRLIDDNEpYTARG were purchased from HysBio Ltd. (Beijing, China). All other chemicals and solvents were of analytical grade. Indium tin oxide conductive glass was supplied by Weiguang Corp. (Shenzhen, P. R. China). The metal complex $\mathrm{Os}(\mathrm{bpy})_{3} \mathrm{Cl}_{2}$ was prepared according to the published procedures. ${ }^{21}$ ITO electrodes (working area $25 \mathrm{~mm}^{2}$ ) were fabricated and cleaned according to the established procedure. ${ }^{8}$

Peptide Damage Reaction (Electrochemical Detection of Peptide Damage). Poly(glu, tyr) $(4: 1)(100 \mathrm{ug} / \mathrm{mL})$ on ITO surface was damaged by reacting with TFBQ in $20 \mathrm{mM}$ phosphate, $\mathrm{pH} 7.4$ buffer solutions. The reaction mixture was incubated at room temperature for the required time, and then the electrode was taken out and rinsed with pure water. Cyclic voltammetry was performed on a CHI 660B electrochemistry analyzer (Austin, TX, USA) with a Pt counter electrode and $3 \mathrm{M} \mathrm{Ag} / \mathrm{AgCl}$ reference electrode, and with 20 $\mathrm{mM}$ phosphate buffer $\mathrm{pH}=7.4$ as supporting electrolyte.

UV-Visible Spectral Analysis of Reaction Products. The interactions between TFBQ and tyrosine were monitored by a UVvisible spectrophotometer (DU-800, Beckman Coulter). The experiment was carried out in the Chelex-treated phosphate buffer $(100 \mathrm{mM}$, $\mathrm{pH}$ 7.4) at room temperature.

HPLC-Q-TOF-MS Analysis. High-performance liquid chromatography (HPLC): A volume of $20 \mu \mathrm{L}$ of each of the reaction mixtures was loaded onto a SUPELCOSIL RP LC- 8 column $(4.6 \times 150 \mathrm{~mm} 3$ $\mu \mathrm{m}$ pore size, Sigma). Elution was performed at $1 \mathrm{~mL} / \mathrm{min}$, starting with $100 \%$ solvent $\mathrm{A}$ ( $1 \%$ acetic acid in water), and the gradient was increased linearly to obtain $94 \%$ methanol in $19 \mathrm{~min}$ and was maintained at $94 \%$ methanol for $1 \mathrm{~min}$, then followed by a linear gradient to obtain $100 \%$ methanol in $25 \mathrm{~min}$. UV detection and recording of UV absorption spectra of the eluted compounds were performed with a 2996 diode array detector ( $2695 \mathrm{XE}$, Waters). The mass spectrometry analysis of the eluted compounds was performed by infusing the HPLC eluents directly into a mass spectrometer (Waters, USA).

MALDI-TOF-MS Analysis. The characterization of peptides and peptide conjugates was performed on an Autoflex III mass spectrometer (Bruker, Germany) both in positive and in negative reflector mode with delayed extraction, typically with an accelerating voltage of $25 \mathrm{kV}$, a grid voltage of $75 \%$, a mirror voltage ratio of 1.12 , a guide wire voltage of $0.05 \%$, and a delay time of $360 \mathrm{~ns}$. Scans were averaged over 100 laser shots, and spectra were baseline-corrected and noise-filtered. The Flexanalysis (version 3.0) software was used for data analysis.

\section{RESULTS AND DISCUSSION}

Tetrafluoro-1,4-benzoquinone (TFBQ) Alone Can Markedly Reduce the Oxidation Current of Tyrosine of Peptide on the Electrochemical Biosensor. In our previous study, an electrochemical biosensor was developed to detect tyrosine and protein oxidation induced by Fenton reagents. ${ }^{20}$ The electrochemical signal of peptide on biosensor decreased remarkably after reaction with Fenton reagents, which are known to produce the highly reactive $\mathrm{HO}^{\bullet}$. When we extended our studies from the classic iron-mediated Fenton system to the new TXBQ-mediated organic Fenton-like system, we found that the oxidation current of peptide decreased to some extent by the combination of tetrafluoro-1,4-benzoquinone (TFBQ) and $\mathrm{H}_{2} \mathrm{O}_{2}$ together as expected. However, unlike in the Fenton system and to our great surprise, the oxidation current decreased remarkably by TFBQ alone (Figure 1),

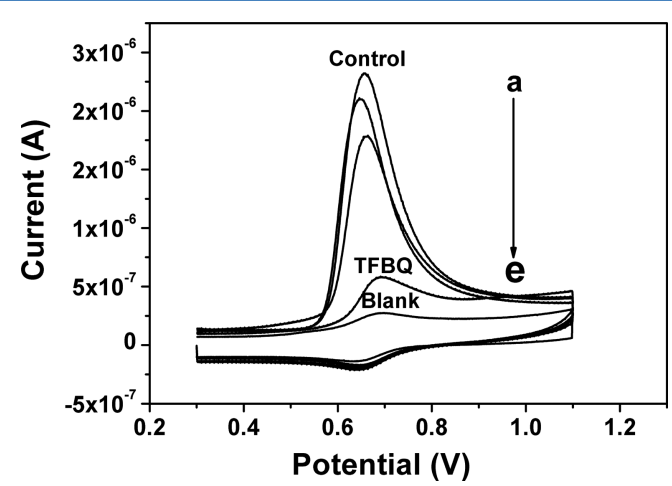

Figure 1. Cyclic voltammograms of poly(glu, tyr) (4:1) coated ITO electrodes in $3 \mu \mathrm{M} \mathrm{Os}(\text { bpy })_{3}{ }^{2+}$ after incubation with (a) $20 \mathrm{mM}$ phosphate buffer ( $\mathrm{pH} 7.4)$, (b) $\mathrm{H}_{2} \mathrm{O}_{2}(500 \mu \mathrm{M})$, (c) TFBQ (100 $\mu \mathrm{M}) / \mathrm{H}_{2} \mathrm{O}_{2}(500 \mu \mathrm{M})$, (d) TFBQ $(100 \mu \mathrm{M})$, and (e) $3 \mu \mathrm{M}$ $\mathrm{Os}(\mathrm{bpy})_{3}^{2+}$.

which reached approximately to the blank control level. The current was found to decrease progressively as TFBQ concentration and reaction time increased, and dropped to the lowest value when the concentration reached $100 \mu \mathrm{M}$ (Figure S1). Analogous results were observed when TFBQ was substituted with other chlorinated quinones, including tetrachloro-, trichloro- and dichloro-1,4-benzoquinones (Figure S2). The effect on electrochemical signal ranks as a decreasing order: $\mathrm{TFBQ}>\mathrm{TCBQ}>\mathrm{TriCBQ} \geq \mathrm{TCHQ}>2,5$-DCBQ. These results suggest that the highly substituted chlorinated quinones are more effective in reacting with tyrosine than the lowly substituted chlorinated benzoquinones.

Among all the halogenated quinones tested, TFBQ was found to react most rapidly and efficiently with peptides and has the most significant effect on the electrochemical signal. Thus, TFBQ was chosen as a model compound for further studies.

The Inhibition of Tyrosine Electroactivity by TFBQ Was Probably Due to the Direct Interactions between the Phenolic Hydroxyl Group of Tyrosine and TFBQ. Since the electroactivity of tyrosine has been attributed to its redox-active hydroxyl group, therefore we speculated that TFBQ may react directly with the hydroxyl group of tyrosine, 
leading to its blockade and loss of its electroactivity. To test this hypothesis, UV-visible spectroscopy was first used to see whether there are any direct interactions between TFBQ and tyrosine. TFBQ has been found to be easily converted to its hydrolyzing product trifluorohydroxy- $p$-benzoquinone (TrFBQ-OH) in buffer solutions. ${ }^{12}$ We found that the absorption intensity of the TrFBQ-OH peak at $525 \mathrm{~nm}$ declined progressively with the increase of tyrosine concentrations, and accompanied by increase of a new intensive peak at $390-400 \mathrm{~nm}$ (Figure S3). These results suggested that a direct interaction may take place between TFBQ (or TrFBQ$\mathrm{OH})$ and tyrosine.

The Modification Was Found To Be Mainly Due to the Formation of a Variety of Fluoroquinone-O-Tyrosine Conjugates and Their Hydroxylated Derivatives via Nucleophilic Substitution. To get a better understanding on the underlying modification mechanisms, the final reaction products between tyrosine and TFBQ were separated and identified by HPLC-ESI-Q-TOF-MS. A series of new peaks were observed in the chromatogram (Figure 2A). It is known

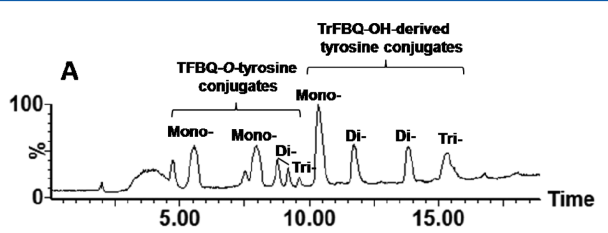

B

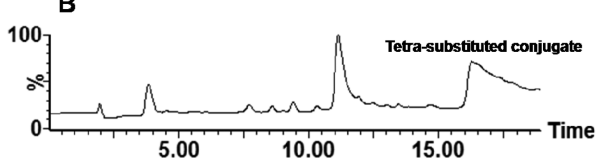

Figure 2. HPLC elution profile of the incubation mixture of (A) $2 \mathrm{mM}$ TFBQ and $2 \mathrm{mM}$ tyrosine and (B) $400 \mu \mathrm{M}$ TFBQ and $2 \mathrm{mM}$ tyrosine in $100 \mathrm{mM}$ ammonium acetate buffer $(\mathrm{pH} \mathrm{7.4)}$ and incubated for 3 min at room temperature. that TXBQs are very good electrophiles, readily reacting with nucleophiles to produce quinone conjugates. ${ }^{8}$ Therefore, we speculated that tyrosine may act as a nucleophile to react with the strong electrophile TFBQ probably via nucleophilic substitution, and found that this is indeed the case.

As shown in Figure 2A, the later-eluting compounds were found to be TFBQ-O-tyrosine conjugates by MS characterization. The highest peak with a retention time of $10.35 \mathrm{~min}$ was identified as monosubstituted TFBQ-O-tyrosine conjugate with loss of a carboxyl group $(m / z=296$, Figure S4). Retention times increased with an increase in the number of fluorines substituted by tyrosine on the benzoquinone. The retention times of disubstituted conjugates were 11.74 and $13.83 \mathrm{~min}$, respectively, indicating that they have two different isomers. Trisubstituted conjugate eluted after disubstituted conjugates at $15.32 \mathrm{~min}$. These conjugates should be formed by successive elimination of one, two, and three fluorine atoms from the quinone ring of TFBQ.

It was found that TrFBQ-OH reacted with tyrosine in a similar fashion as TFBQ, with the formation of analogous products (Figure S5). Interestingly, we found that the mono(5.56 and $7.97 \mathrm{~min}$ ) and disubstituted (8.77 and $9.18 \mathrm{~min}$ ) conjugates both have two different isomers which have very close retention times because of their close similarities in structure, polarity, and charged state.

When we increased the molar ratio of TFBQ:tyrosine to $1: 5$, a new broad peak with the retention time at 16.2 min was observed (Figure 2B). This new peak was identified as tetrasubstituted conjugate by MS, with displacement of all four fluorine with four tyrosyl groups. These results indicate that TFBQ should be more ready to react with tyrosine instead of being hydrolyzed to TrFBQ-OH when tyrosine is in excess. Thus, TFBQ would retain its oxidized quinone structure after reacting with one molecule of tyrosine and continues to react with additional tyrosine molecules consecutively until all four fluorine atoms have been substituted. Consequently, the peaks

Scheme 1. Proposed Reaction Pathways for the Reaction between TFBQ and Tyrosine in $100 \mathrm{mM}$ Ammonium Acetate Buffer (pH 7.4) and Incubated for $3 \mathrm{~min}$ at Room Temperature

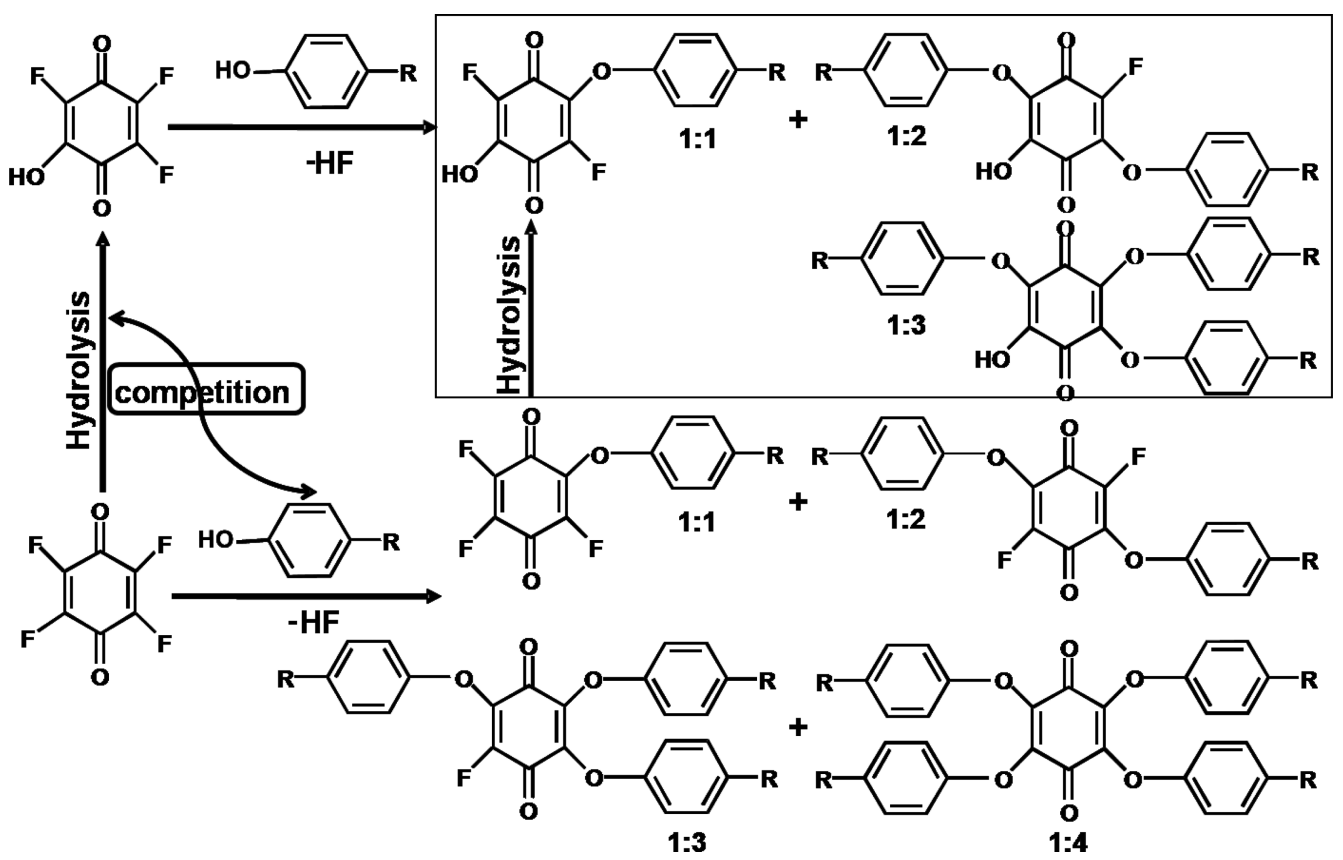



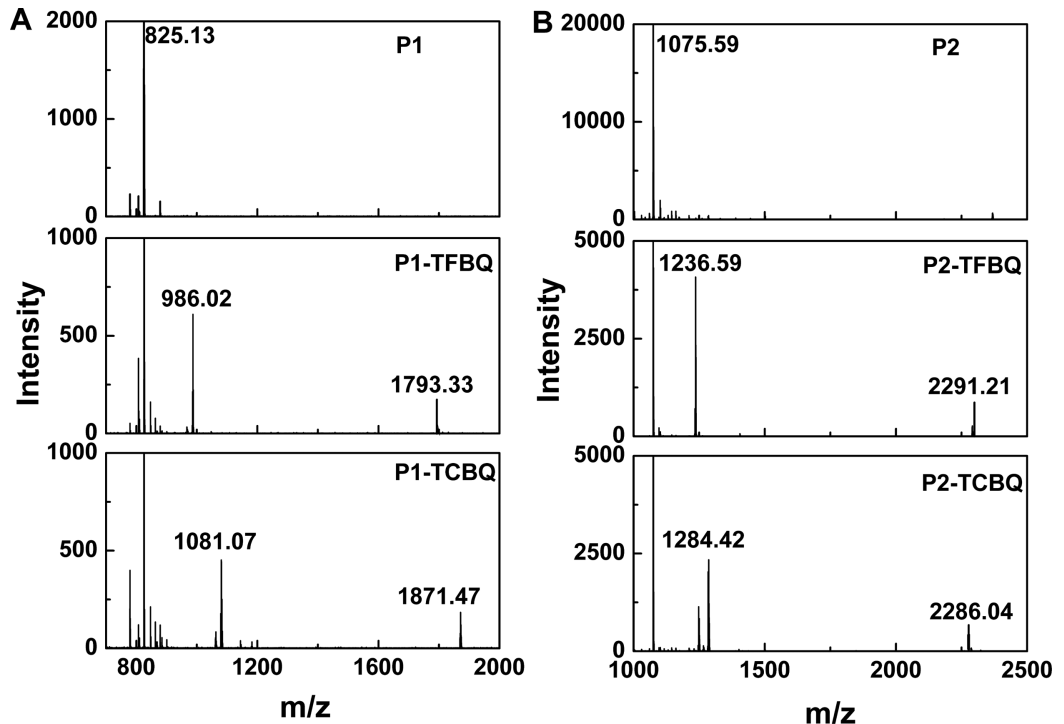

Figure 3. Mass spectra of peptide $(300 \mu \mathrm{M})$ control and incubated mixtures of (A) P1 and (B) P2 with TXBQs (100 $\mu \mathrm{M})$ showing the formation of quinone-tyrosine-containing peptide conjugates.

of a series of TFBQ-O-tyrosine conjugates and TrFBQ-OHderived tyrosine conjugates became much smaller.

For both TFBQ- and TrFBQ-OH-derived tyrosine conjugates, integrated areas of chromatographic peaks show that the levels of these conjugates were in the following order: mono- $>$ di- $>$ trisubstituted. The decrease in level of these conjugates indicates that the highly substituted conjugates might be more difficult to be produced, possibly due to steric hindrance effect of the bulky tyrosyl group. The level of all conjugates is time-dependent because TFBQ is very reactive in aqueous buffer solution. We found that the level of TrFBQ$\mathrm{OH}$-derived tyrosine conjugates increased with time, accompanied by concurrent decrease of TFBQ-tyrosine conjugates, indicating that TFBQ-derived tyrosine conjugates are not stable and can be easily converted to their corresponding hydrolysis product.

In order to further confirm the structure of these conjugates, MS-MS spectra of these eluted conjugates were collected (Figures S6, S7, S8, S9). We found that most of the parent ions were fragmented with the subsequent loss of carboxyl group $(\mathrm{m} / z 46)$ and ammonium group $(\mathrm{m} / z 17)$ of amino acid. The comparison of the MS-MS spectra of the same substituted TFBQ- and TrFBQ-OH-derived tyrosine conjugates indicated that they may undergo a similar fragmentation pathway. These MS-MS results provided stronger experimental evidence for the proposed structures of these quinone-O-tyrosine conjugates.

Compared to TFBQ, we found that only a small amount of the lowly substituted (such as mono- and disubstituted), but not the highly substituted (such as tri- and tetrasubstituted) TCBQ-derived tyrosine conjugates, were produced (Figures S10, S11). The quantity of TCBQ-derived tyrosine conjugates was found to be lower than that of the corresponding TFBQtyrosine conjugates. Additionally, it takes a longer time for TCBQ to react with tyrosine completely than that of TFBQ. These results suggest that TCBQ is much less reactive in reacting with tyrosine than TFBQ. In contrast, no TrCBQ-OH (trichlorohydroxy-1,4-benzoquinone)-derived tyrosine conjugates were observed, which is possibly due to much slower hydrolysis rate of TCBQ to $\mathrm{TrCBQ}-\mathrm{OH}$, and much lower reactivity of $\mathrm{TrCBQ}-\mathrm{OH} .^{22}$

Molecular Mechanism for the Modification of the Phenolic Hydroxyl Group of Tyrosine by TFBQ. Based on the above experimental results, we proposed the following molecular mechanism for the modification of the phenolic hydroxyl group of tyrosine by TFBQ (Scheme 1). A nucleophilic substitution reaction may take place between TFBQ and tyrosine, forming different multisubstituted conjugates by successive substitution of fluorine atom by tyrosine from the quinone ring of TFBQ. These conjugates can further be converted to their hydroxylated derivatives. However, when tyrosine is in excess, higher-substituted conjugates are the major products. Alternatively, TFBQ can also readily be hydrolyzed to $\operatorname{TrFBQ}-\mathrm{OH}$, which further reacted with tyrosine to form TrFBQ-OH-derived O-tyrosine conjugates. The two reaction pathways are competitive, and the main products should be dependent on the molar ratios of reactants and the reaction time. To our knowledge, this is the first report showing that the tyrosine phenoxyl group could be readily modified by TFBQ via nucleophilic substitution under normal physiological conditions.

Modification of the Phenolic Hydroxyl Group of Tyrosine-Containing peptides by TFBQ: Characterization of Quinone-O-Tyrosine-Containing Peptide Conjugates by MALDI-TOF-MS Analysis. It should be interesting to know whether our finding is not only limited to tyrosine but also applies to more general tyrosine-containing peptides. For this purpose, two tyrosine-containing peptides with different sequences were chosen as model peptides (P1, EEEEEY; P2, AGYTAIVSHR). P1 is one of the subunits of poly(glu, tyr) peptide on the electrochemical biosensor, thus the interaction between $\mathrm{P} 1$ and TXBQ can further confirm the reaction between the phenoxyl group of tyrosine-containing polypeptide and TFBQ. Matrix-assisted laser desorption/ ionization mass spectrometry (MALDI-TOF-MS) was used to characterize the reaction products.

As shown in Figure 3A, two new protonated molecules at $\mathrm{m} /$ $z 986.02$ and 1793.3 were detected after incubation of TFBQ with P1. We speculate that the protonated molecule at $\mathrm{m} / \mathrm{z}$ 
986.02 corresponds to the conjugate of TFBQ with one P1, and the other one at $m / z 1793.3$ represents the conjugate of TFBQ with two P1. These results suggested that TFBQ reacted with free phenoxyl groups of the peptide to form two substituted conjugates. Similar mono- and disubstituted conjugates of TFBQ with P2 were also detected (Figure 3B). Analogous results were obtained when TFBQ was displaced by TCBQ. The mono- and disubstituted conjugates of TCBQ with peptides were characterized by the unique three- and twochlorine isotope cluster, respectively, in the MS spectrum.

To identify the modification sites on both peptides, the conjugates were further characterized by MS/MS. The protonated molecule with $\mathrm{m} / z 963$ corresponds to the disubstituted conjugate of P1-TrFBQ-OH with loss of one molecule P1. The MS/MS spectrum of precursor ion $\mathrm{m} / z 963$ gave a series of $y$ - $(447,576,705)$ and b-series $(128,239)$ fragment ions, confirming that the $\mathrm{N}$-terminal tyrosine residue is the addition site of TrFBQ-OH (Figure S13A). The MS/MS spectrum of precursor ion $\mathrm{m} / z 1035$ (Figure S13B) also contained the characteristic peak $y$ - of TCBQ-tyrosine residue conjugate. Meanwhile, the MS/MS spectrum of TXBQ with P2 also matched well with the expected fragment ions. These MS/ MS results further confirmed that tyrosine residue is indeed the modification site by TXBQ.

Inhibition of Tyrosine Phosphorylation by TXBQs. Protein tyrosine phosphorylation is achieved by transfer of $\gamma$ phosphate group from ATP to the phenoxyl group of tyrosine in protein through the catalysis of protein tyrosine kinases (PTKs). ${ }^{23}$ Based on the above new findings that TXBQ can modify the $\mathrm{OH}$ group on tyrosine via forming quinone-Otyrosine conjugates, we expect that the substrate peptide modified by TXBQs should not be able to bind to PTK and the tyrosine phosphorylation should be prevented. To test the above hypothesis, a synthetic peptide RRLIEDNEYTARG was chosen as the substrate peptide and epidermal growth factor receptor (EGFR) as a model PTK. The effect of TXBQs on the catalytic activities of EGFR toward substrate peptide was investigated following the established method. ${ }^{24}$

First we investigated whether the conjugates could be formed between substrate peptide and TXBQs by MS, and found that this is indeed the case. The quantity of phosphorylated substrate peptide was found to be decreased sharply after preincubation of the substrate peptide $(36 \mu \mathrm{M})$ with TFBQ indicating that TFBQ inhibited tyrosine phosphorylation in a concentration-dependent mode (Figure 4). Fifty percent

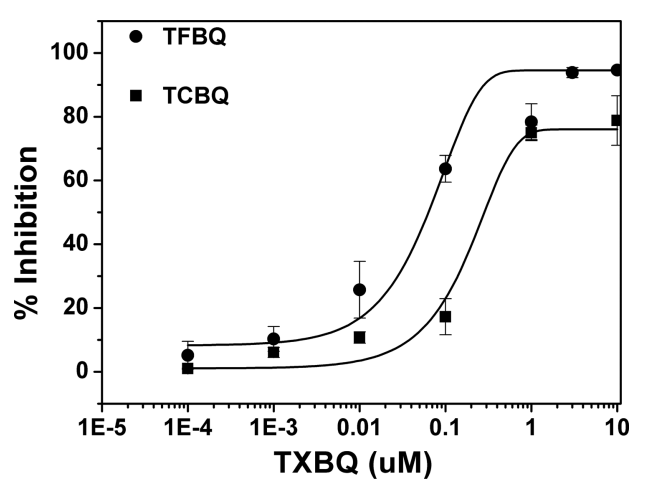

Figure 4. Dose-dependent inhibition curves of TFBQ (circle) and TCBQ (square) on phosphorylation of peptide RRLIEDNEYTARG generated by MALDI-TOF-MS analysis. inhibition of tyrosine phosphorylation can be achieved when TFBQ concentration $\left(\mathrm{IC}_{50}\right)$ was only $50 \mathrm{nM}$, which is much lower than that of TCBQ $\left(\mathrm{IC}_{50}: 400 \mathrm{nM}\right)$. These results proved that TFBQ and TCBQ can inhibit the tyrosine phosphorylation efficiently under normal physiological conditions because they can block the phenoxyl group of tyrosine residue, which further supported the above proposed reaction mechanism.

Potential Biological and Environmental Implications. Our observations that not only TFBQ and TCBQ but also other chlorinated quinones can react with tyrosine via nucleophilic substitution may have potential biological and environmental implications: Unlike the synthetic chemical modification reagents, TXBQs are naturally occurring metabolites of many widely used polyhalogenated aromatic compounds including polyhalogenated phenols (such as the ubiquitous pentachlorophenol), Agent Orange, hexachlorobenzene, and polychlorinated biphenyls. ${ }^{25-27}$ Recently several polyhalogenated quinones were also identified as disinfection byproducts in drinking and swimming pool water, which are suspected bladder carcinogens. ${ }^{28}$ It is well-known that the toxicity of quinone metabolites is mainly through their swift covalent binding to thiol groups. ${ }^{8,13}$ Here our findings that TXBQs can modify tyrosine with high reactivity may provide some unexpected new insight into their potential toxicity. Taking into consideration the importance of phenoxyl group of tyrosine residues in many proteins and the availability of TXBQs in biological systems, our findings may partly explain the toxic effects of the halogenated aromatic environmental pollutants.

In addition, chemical modification on individual amino acids has proven to be a useful tool for the investigation of protein structure-function relationships. In this regard, due to its high efficiency and mild reaction conditions for the interactions between TXBQs and tyrosine, the present study was undertaken in an attempt to probe the roles of tyrosine residue in protein function. For example, modification of some enzymes which contain tyrosine residue as their active sites by TXBQs may probably render the loss of protein function. It has been reported that glutathione conjugate with TCBQ might irreversibly inhibit the glutathione $S$-transferase isoenzyme by modification of two active-site tyrosyl residues by computational studies. ${ }^{29}$ Although some evidence has been provided that tyrosine might be the potential target of quinone conjugate in their studies, the underlying precise molecular mechanism is still unclear. Therefore, our data may provide partial experimental support for their presumption.

The results presented in this study clearly elucidate the detailed reaction mechanism between tyrosine and TXBQs and strongly suggest that tyrosine is a feasible modification site by polyhalogenated quinones in addition to cysteine residue in protein. However, it should be noted that our study is only limited to tyrosine and tyrosine-containing peptides. Thus, further investigations are needed to study whether these reactions could occur on other proteins and are relevant in cell culture system or in vivo.

\section{CONCLUSIONS}

In conclusion, we found that TXBQs can react with tyrosine and tyrosine-containing peptides to form different substituted conjugates via nucleophilic substitution. Chemical modification of the phenoxyl group of tyrosine by TXBQs can prevent tyrosine phosphorylation in substrate peptide, which should have some influence on the protein function and the 
subsequent cell signal transduction in biological systems. This represents the first report showing that the phenoxyl group of tyrosine can be readily modified by TXBQs under normal physiological conditions, which may partly explain the strong toxicity for halogenated quinones and their parent polyhalogenated aromatic environmental pollutants.

\section{ASSOCIATED CONTENT}

\section{S Supporting Information}

The Supporting Information is available free of charge on the ACS Publications website at DOI: 10.1021/acs.chemrestox.6b00217.

Oxidation current responses, UV-vis, MS, and MS/MS spectra, and HPLC profile (PDF)

\section{AUTHOR INFORMATION}

\section{Corresponding Author}

*E-mail: bzhu@rcees.ac.cn.

\section{Funding}

The work in this paper was supported by the Strategic Priority Research Program of CAS Grant No. XDB01020300; NSF China grants (21207149, 21207139, 21477139, 21237005, and 21321004); and NIH grants (ES11497, RR01008, and ES00210).

\section{Notes}

The authors declare no competing financial interest.

\section{ACKNOWLEDGMENTS}

We thank Dr. Dong Cao for his assistance with the MALDITOF-MS experiments.

\section{ABBREVIATIONS}

TXBQs, tetrahalo-1,4-benzoquinones; TFBQ tetrafluoro-1,4benzoquinone; TCBQ, tetrachloro-1,4-benzoquinone; TCHQ, tetrachloro-1,4-hydroquinone; $\operatorname{TrCBQ}$ trichloro-1,4-benzoquinone; DCBQ, dichloro-1,4-benzoquinones; TrFBQ-OH, trifluorohydroxy-p-benzoquinone; TrCBQ-OH, trichlorohydroxy1,4-benzoquinone; POPs, polychlorinated persistent organic pollutants; PTMs, post-translational modifications; PTKs, protein tyrosine kinases; EGFR, epidermal growth factor receptor; ATP, adenosine triphosphate; ITO, indium tin oxide; PDDA, poly(diallyldimethylammonium chloride); HPLC, high-performance liquid chromatography; UV, ultraviolet; MALDI-TOF-MS, matrix-assisted laser desorption/ ionization mass spectrometry

\section{REFERENCES}

(1) Souza, J. M., Peluffo, G., and Radi, R. (2008) Protein tyrosine nitration-functional alteration or just a biomarker? Free Radical Biol. Med. 45, 357-366.

(2) Stone, M. J., Chuang, S., Hou, X., Shoham, M., and Zhu, J. Z. (2009) Tyrosine sulfation: an increasingly recognised post-translational modification of secreted proteins. New Biotechnol. 25, 299-317.

(3) Hubbard, S. R., and Till, J. H. (2000) Protein tyrosine kinase structure and function. Annu. Rev. Biochem. 69, 373-98.

(4) Baroni, S., Pandini, V., Vanoni, M. A., and Aliverti, A. (2012) A single tyrosine hydroxyl group almost entirely controls the NADPH specificity of plasmodium falciparum Ferredoxin-NADP ${ }^{+}$reductase. Biochemistry 51, 3819-3826.

(5) Gao, R., Zhang, Y., Dedkova, L., Choudhury, A. K., Rahier, N. J., and Hecht, S. M. (2006) Effects of modification of the active site tyrosine of human DNA topoisomerase I. Biochemistry 45, 8402-8410.
(6) Gao, R., Zhang, Y., Choudhury, A. K., Dedkova, L. M., and Hecht, S. M. (2005) Analogues of vaccinia virus DNA topoisomerase I modified at the active site tyrosine. J. Am. Chem. Soc. 127, 3321-3331.

(7) Riordan, J. F., Wacker, W. E. C., and Vallee, B. L. (1965) NAcetylimidazole: A reagent for determination of "Free" tyrosyl residues of proteins. Biochemistry 4, 1758-1765.

(8) Waidyanatha, S., Lin, P. H., and Rappaport, S. M. (1996) Characterization of chlorinated adducts of hemoglobin and albumin following administration of pentachlorophenol to rats. Chem. Res. Toxicol. 9, 647-653.

(9) Tsai, C. H., Lin, P. H., Troester, M. A., and Rappaport, S. M. (2003) Formation and removal of pentachlorophenol-derived protein adducts in rodent liver under acute, multiple, and chronic dosing regimens. Toxicol. Sci. 73, 26-35.

(10) Zhu, B.-Z., and Shan, G.-Q. (2009) Potential mechanism for pentachlorophenol-induced carcinogenicity: a novel mechanism for metal-independent production of hydroxyl radicals. Chem. Res. Toxicol. 22, 969-977.

(11) den Besten, C., van Bladeren, P. J., Duizer, E., Vervoort, J., and Rietjens, I. M.C.M. (1993) Cytochrome P450-mediated oxidation of pentafluorophenol to tetrafluorobenzoquinone as the primary reaction product. Chem. Res. Toxicol. 6, 674-680.

(12) Osman, A. M., Posthumus, M. A., Veeger, C., van Bladeren, P. J., Laane, C., and Rietjens, I. M. C. M. (1998) Conversion of pentahalogenated phenols by microperoxidase- $8 / \mathrm{H} 2 \mathrm{O} 2$ to benzoquinone-type products. Chem. Res. Toxicol. 11, 1319-1325.

(13) Waidyanatha, S., McDonald, T. A., Lin, P.-H., and Rappaport, S. M. (1994) Measurement of hemoglobin and albumin adducts of tetrachlorobenzoquinone. Chem. Res. Toxicol. 7, 463-468.

(14) Zhu, B. Z., Kalyanaraman, B., and Jiang, G. B. (2007) Molecular Mechanism for metal-independent production of hydroxyl radicals by hydrogen peroxide and halogenated quinones. Proc. Natl. Acad. Sci. U. S. A. 104, 17575-17578.

(15) Zhu, B.-Z., Antholine, W. E., and Frei, B. (2002) Thiourea protects against copper-induced oxidative damage by formation of a redox-inactive thiourea-copper complex. Free Radical Biol. Med. 32, $1333-1338$.

(16) Zhu, B.-Z., Shan, G. Q., Huang, C. H., Kalyanaraman, B., Mao, L., and Du, Y. G. (2009) Metal-independent decomposition of hydroperoxides by halogenated quinones: Detection and identification of a quinone ketoxy radical. Proc. Natl. Acad. Sci. U. S. A. 106, 1146611471.

(17) Shao, J., Huang, C. H., Kalyanaraman, B., and Zhu, B.-Z. (2013) Potent methyl oxidation of 5-methyl-2'-deoxycytidine by halogenated quinoid carcinogens and hydrogen peroxide via a metal-independent mechanism. Free Radical Biol. Med. 60, 177-182.

(18) Zhao, B. L., Yang, Y., Wang, X. L., Chong, Z. C., Yin, R. C., Song, S.-H., Zhao, C., Li, C. P., Huang, H., Sun, B.-F., Wu, D. N., Jin, K.-X., Song, M. Y., Zhu, B.-Z., Jiang, G. B., Rendtlew Danielsen, J. M., $\mathrm{Xu}, \mathrm{G}$. L., Yang, Y.-G., and Wang, H. L. (2014) Redox-active quinones induces genome-wide DNA methylation changes by an iron-mediated and Tet-dependent mechanism. Nucleic Acids Res. 42, 1593-1605.

(19) Yin, R. C., Zhang, D. P., Song, Y. L., Zhu, B.-Z., and Wang, H. L. (2013) Potent DNA damage by polyhalogenated quinones and $\mathrm{H} 2 \mathrm{O} 2$ via a metal-independent and Intercalation-enhanced oxidation Mechanism. Sci. Rep. 3, 1269.

(20) Qu, N., Guo, L.-H., and Zhu, B.-Z. (2011) An electrochemical biosensor for the detection of tyrosine oxidation induced by Fenton reaction. Biosens. Bioelectron. 26, 2292-2296.

(21) Hamann, T. W., Gstrein, F., Brunschwig, B. S., and Lewis, N. S. (2005) Measurement of the free-energy dependence of interfacial charge-transfer rate constants using $\mathrm{ZnO} / \mathrm{H}_{2} \mathrm{O}$ Semiconductor/Liquid Contacts. J. Am. Chem. Soc. 127, 7815-7824.

(22) Zhu, B.-Z., Har-El, R., Kitrossky, N., and Chevion, M. (1998) New modes of action of desferrioxamine: Scavenging of semiquinone radical and stimulation of hydrolysis of tetrachlorohydroquinone. Free Radical Biol. Med. 24, 360-369.

(23) Hunter, T. (1995) Protein Kinases and Phosphatases: The Yin and Yang of protein phosphorylation and signaling. Cell 80, 225-236. 
(24) Ji, L. Y., Wu, J.-H., Luo, Q., Li, X. C., Zheng, W., Zhai, G. J., Wang, F. Y., Lü, S., Feng, Y.-Q., Liu, J. A., and Xiong, S. X. (2012) Quantitative mass spectrometry combined with separation and enrichment of phosphopeptides by titania coated magnetic mesoporous silica microspheres for screening of protein kinase inhibitors. Anal. Chem. 84, 2284-2291.

(25) Song, Y., Wagner, B. A., Witmer, J. R., Lehmler, H. J., and Buettner, G. R. (2009) Nonenzymatic displacement of chlorine and formation of free radicals upon the reaction of glutathione with $\mathrm{PCB}$ quinones. Proc. Natl. Acad. Sci. U. S. A. 106, 9725-9730.

(26) Van Ommen, B., Adang, A. E. P., Brader, L., Posthumus, M. A., Müller, F., and Van Bladeren, P. J. (1986) The microsomal metabolism of hexachlorobenzene: Origin of the covalent binding to protein. Biochem. Pharmacol. 35, 3233-3238.

(27) Kelly, B. C., Ikonomou, M. G., Blair, J. D., Morin, A. E., and Gobas, F. A. (2007) Food web-specific biomagnification of persistent organic pollutants. Science 317, 236-239.

(28) Li, J. H., Wang, W., Moe, B., Wang, H. L., and Li, X.-F. (2015) Chemical and toxicological characterization of halobenzoquinones, an emerging class of disinfection byproducts. Chem. Res. Toxicol. 28, 306318.

(29) Jan, H. T. M., Ploemen, S., Johnson, W. W., Jespersenn, S., Vanderwalls, D., Ommen, B. V., Green, J. V., Bladeren, P. V. S., and Armstrong, R. N. (1994) Active-site tyrosyl residues are targets in the irreversible inhibition of a class $\mathrm{Mu}$ glutathione transferase by 2-(Sglutathionyl)-3,5,6-trichloro-1,4-benzoquinone. J. Biol. Chem. 269, 26890-26897. 